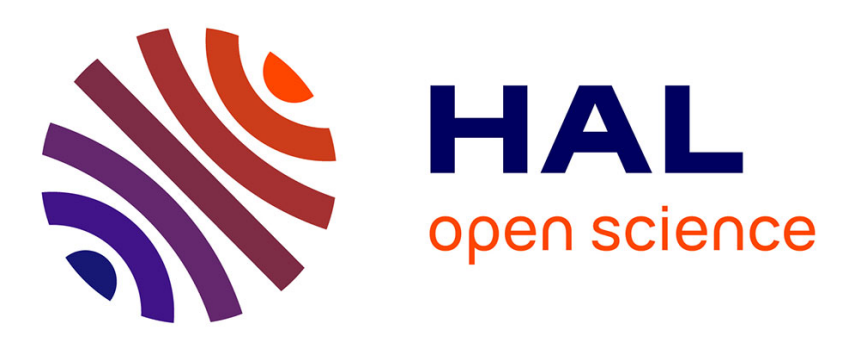

\title{
Irrigation Optimization by Modeling of Plant-Soil Interaction
}

Zhongping Li, Paul-Henry Cournède, Jean-Claude Mailhol

\section{To cite this version:}

Zhongping Li, Paul-Henry Cournède, Jean-Claude Mailhol. Irrigation Optimization by Modeling of Plant-Soil Interaction. 2011. hal-00606408

\section{HAL Id: hal-00606408 https://hal.science/hal-00606408}

Preprint submitted on 6 Jul 2011

HAL is a multi-disciplinary open access archive for the deposit and dissemination of scientific research documents, whether they are published or not. The documents may come from teaching and research institutions in France or abroad, or from public or private research centers.
L'archive ouverte pluridisciplinaire HAL, est destinée au dépôt et à la diffusion de documents scientifiques de niveau recherche, publiés ou non, émanant des établissements d'enseignement et de recherche français ou étrangers, des laboratoires publics ou privés. 


\section{IRRIGATION OPTIMIZATION BY MODELING OF PLANT-SOIL INTERACTION}

\author{
Zhongping Li \\ Laboratory of Applied Maths and Systems \\ Ecole Centrale Paris \\ Châtenay-Malabry, France \\ zhongping.li@ecp.fr
}

\author{
Paul-Henry Cournède \\ Laboratory of Applied Maths and Systems \\ Ecole Centrale Paris \\ Châtenay-Malabry, France
}

\author{
Jean-Claude Mailhol \\ UMR G-EAU \\ Cemagref \\ Montpellier, France
}

\begin{abstract}
Irrigation scheduling is an important issue for crop management, in a general context of limited water resources and increasing concern about agricultural productivity. Methods to optimize crop irrigation should take into account the impact of water stress on plant growth and the water balance in the plant-soil-atmosphere system. In this article, we propose a methodology to solve the irrigation scheduling problem. For this purpose, a plant-soil interaction model is used to simulate the structural-functional plant growth conditioned by water status. The system dynamics is driven by a delay differential system. By considering a price for the crop yield and for the water resource, an optimal control problem can be formulated in order to find the optimal irrigation strategy. The solution is obtained by dynamic programming. In order to handle the delay term of the system due to the continuous mechanism of plant senescence and the curse of dimensionality, the iterative approach of dynamic programming is used.
\end{abstract}

\section{KEY WORDS}

Irrigation scheduling, plant-soil model, water stress, delay differential system, iterative dynamic programming

\section{Introduction}

The effective use of water for agriculture is a crucial priority for farmers. A lot of research and investments have been made to develop more refined techniques and practices to apply water accurately to crops according to their requirements. Most irrigation scheduling methods use measured or estimated soil moisture status as criterion to determine when and how much water to apply to the field ([9]). In this article, we propose an alternative approach of irrigation scheduling based on the plant-soil interaction modeling, and with criterion to maximize the final net return.

To explore the plant-soil interaction, two crop models GreenLab and PILOTE are coupled. GreenLab is a functional-structural model which gives a fine description of plant growth at organ level ([4]). PILOTE predicts empirically crop yield according to the Leaf Area Index (LAI) evolution, where LAI is adjusted by a water stress index computed with a submodel of soil water balance ([18], [10]).
In the coupling approach, we simulate the soil water balance by PILOTE to get the water stress index. Plant responses to water stress involve diverse physiological processes ([6], [7]). The strongest impact is on the radiation use efficiency driving the dry matter accumulation. It needs to be adjusted according to the water stress index, as global functional result. This may be seen as an improvement of GreenLab with respect to water status consideration. At the same time, we use the LAI simulated mechanically by GreenLab when calculating evapotranspiration in the update of soil water balance.

The dynamic of this plant-soil model is described by a differential equation system, with delay term due to the continuous senescence mechanism of plant detailed in [13].

Such a complete description of plant-soil interaction formulated as a dynamic system enables the application of mathematical optimization methods. In particular, the dynamic programming is well adapted here to generate a optimal irrigation policy.

However, the high dimensionality makes difficult a direct implementation of dynamic programming to the system, and we also need to adapt the algorithm to the delay term. For these purposes, the iterative version of dynamic programming is introduced ([15]). In each iteration, the delay term is approximated by using precaculated trajectories. These trajectories as well as the grid for state and control variables are generated coarsely. Finally, the computation of the global optimal solution is based on the systematic region contraction at each iteration and the convergence of solutions by iteration.

\section{Model description}

In this section, We present the GreenLab model for the plant functional growth as well as the soil water balance submodel of PILOTE, and we show how they can be coupled in a plant-soil model.

\subsection{GreenLab}

To describe the functional plant development, we use the continuous version of GreenLab model which is suitable for integrating environmental variables([13]). The choice 
of time unit is crucial to describe the interaction of plant growth with the environment. The environmental variations are better described as functions of the calendar time, while plant development and expansion are closely related to the thermal time ([16]), defined as

$$
\tau(t)=\int_{0}^{t} \max \left(0, T(s)-T_{b}\right) d s,
$$

with $T(s)$ the temperature at calendar time $s, T_{b}$ the base temperature and $t=0$ at growth initiation.

\subsubsection{Organogenesis and demand}

The architectural development of plant is described as a sequence of growth unit appearances as well as the expansion of the organs constituting the growth units ([4],[2]). Generally for crops, the thermal time elapsing between successive appearances of phytomers can be considered as constant and is called phyllochron ([23]). It is denoted by $\gamma$ and is regarded as the growth cycle in GreenLab. In the following, we consider that organogenesis events occur at integer multiples of $\gamma$.

Biomass partitioning is made according to a simple source-sink model. Every organ is regarded as a sink whose strength depends on the type of organ and varies according to its thermal age during the period of its expansion, denoted by $\tau_{o} . o$ may stand for blade, petiole, internode... and we denote $O$ for the set of all the organ types constituting a specific plant. The biomass produced by photosynthesis (or provided by the seed before plant emergence) is allocated to the organs proportionally to the strength of each $\operatorname{sink}([25])$.

In GreenLab, the demand of an organ of type $o$ (noting that the root system is considered as a single pool) at its thermal age $u$ is

$$
\mathrm{P}_{o}(u)= \begin{cases}p_{o} f_{a_{o}, b_{o}}(u) & \text { for } 0 \leq u \leq \tau_{o} \\ 0 & \text { otherwise }\end{cases}
$$

with $p_{o}$ the organ base sink strength, and $f_{a_{o}, b_{o}}$ is the normalized beta function defined as

$$
f_{a_{o}, b_{o}}(u)=\frac{1}{M_{o}}\left(\frac{u}{\tau_{o}}\right)^{a_{o}-1}\left(1-\frac{u}{\tau_{o}}\right)^{b_{o}-1}
$$

with $M_{o}=\sup _{u \in\left[0, \tau_{o}\right]}\left(\frac{u}{\tau_{o}}\right)^{a_{o}-1}\left(1-\frac{u}{\tau_{o}}\right)^{b_{o}-1}$.

Therefore, when plant has thermal age $u(u=0$ at plant's initiation), the demand of all the organs of type $o$ is

$$
P_{o}(u)=\sum_{k \in \mathbb{N}} N_{o}(k) \mathrm{P}_{o}(u-k \gamma),
$$

where $N_{o}(k)$ denotes the number of organs given by organogenesis model (see [2]). and we denote $D(u)=$ $\sum_{o \in O} P_{o}(u)$ for the total demand of the plant.

\subsubsection{The initial phase}

We assume that the seed distributes its mass uniformly from plant's initiation till its emergence (visual appearance of the first phytomer) to organs of the first growth unit, therefore

$$
Q_{b}(t)=\int_{0}^{\tau(t)} \frac{Q^{\text {seed }}}{\gamma} \frac{P_{b}(u)}{D(u)} d u,
$$

for $t \in\left[0, \tau^{-1}(\gamma)\left[, Q^{\text {seed }}\right.\right.$ denoting the seed mass.

\subsubsection{Production}

Biomass production by photosynthesis depends on the growth stage, on the available incident energy (radiation), and on resources status (here limited to water).

In order to underline the coupling character with the hydric model, the biomass production according to GreenLab ([12]) is adjusted by the stress function $f_{s}$ (see eq.19). Thus, we have for the rate of biomass production

$$
q(t)=\mu d \operatorname{PAR}(t) S_{p}\left(1-\exp \left(-k \frac{S(t)}{S_{p}}\right)\right) f_{s}(t)
$$

$\mu$ is the empirical coefficient related to the radiation use efficiency, assumed to equal 0.48 times the global incident radiation ([22]). $\operatorname{PAR}(t)$ denotes the incident photosynthetically active radiation accumulated since plant emergence till $t, k$ the Beer-Lambert extinction coefficient and $S_{p}$ the empirical coefficient corresponding to a characteristic surface. Notice that $\mu, S_{p}$ are parameters specific to a given crop and growing conditions. And $S(t)$ is the total leaf surface area of the plant at time $t$.

Making the assumption that leaves have a constant mass per unit area $e$, the total leaf surface can be written as:

$$
S(t)=\frac{Q_{b}(t)}{e}
$$

Foliar mass $Q_{b}$ is calculated by taking into account its senescence. For the sake of simplicity, we suppose that all leaves have the same longevity $\tau^{\text {sen }}$ in thermal time. Therefore, the part becoming senescent at time $t$ equals to the part allocated to leaves at time $\eta(t)=\tau^{-1}\left(\tau(t)-\tau^{\text {sen }}\right)$ ([14]). Thus the variation of foliar mass is given by:

$$
d Q_{b}(t)=f\left(t, Q_{b}(t)\right)-f\left(\eta(t), Q_{b}(\eta(t))\right)
$$

where:

$$
f\left(t, Q_{b}(t)\right)=\frac{P_{b}(\tau(t))}{D(\tau(t))} q(t)
$$

For the computation of soil water balance, we need the amount of water transpirated by plant on a surface of $1 m^{2}$. This quantity varies according to the LAI, that is expressed as:

$$
L A I(t)=S(t) \cdot d
$$

with $d$ the field density. 


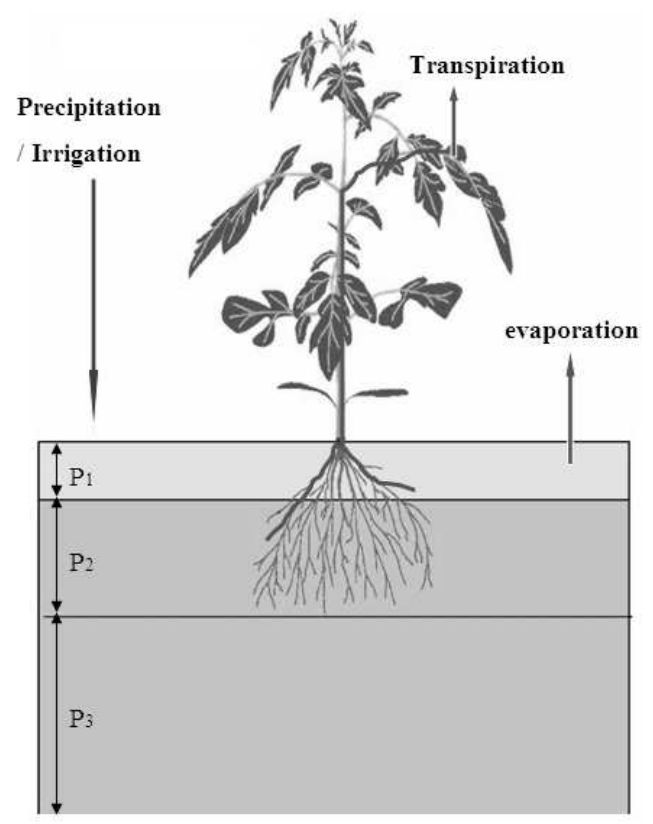

Figure 1. The three reservoirs and the soil water balance in PILOTE

\subsection{PILOTE}

PILOTE is a crop model predicting yield values by using an empirical LAI curve, and by simulating a soil water balance from which we may extract a indicator of water stress for the plant ([17],[10]). In the coupled model, we use this stress indicator in the calculation of dry mass production (see eq.6). Meanwhile, when simulating the soil water balance, instead of the empirical value of LAI from PILOTE, we use the LAI simulated dynamically by GreenLab.

\subsubsection{Reservoirs}

The soil module is divided into 3 reservoirs with respective depths $P_{1}$ (fixed, corresponding to the superficial soil layer), $P_{2}(t)=P_{r}(t)-P_{1}, P_{3}(t)=P_{X}-P_{2}(t)-P_{1}$. Where $P_{r}(t)$ is the current root depth, $P_{X}$ the maximal root depth (see Fig.1).

The three reservoirs have the same minimum and the maximal volumetric soil water content $\Theta_{\min }, \Theta_{\max }$. And soil water content available to the plant in reservoir $i$ is:

$$
R_{i}(t)=\left(\Theta_{i}(t)-\Theta_{\min }\right) P_{i}(t)
$$

with $\Theta_{i}$ the volumetric soil water content of reservoir $i$, we note also $R_{i \max }(t)=\left(\Theta_{\max }-\Theta_{\min }\right) P_{i}(t)$. The water balance of these reservoirs takes into account precipitation $P$ (for $R_{1}$ ), irrigation $I$ (for $R_{1}$ ), and plant transpiration $T p$ (for $R_{1}, R_{2}$ ), soil evaporation $E s$ (for $R_{1}$ ), and drainage $d_{i}$ (for $R_{1}, R_{2}, R_{3}$ ). Thus,

$$
\begin{aligned}
& d R_{1}(t)=P(t)+I(t)-T p_{1}(t)-E s(t)-d_{1}(t) \\
& d R_{2}(t)=d_{1}(t)+\Theta_{3}(t) V_{r}-T p_{2}(t)-d_{2}(t) \\
& d R_{3}(t)=d_{2}(t)-\Theta_{3}(t) V_{r}-d_{3}(t)
\end{aligned}
$$

where $V_{r}$ is the root growth rate supposed to be constant.

\subsubsection{Evapotranspiration in $R_{1}$}

When $R_{1}(t)>0$, plant transpiration takes place only in $R_{1}$. We denote $T p_{1 \text { max }}$ the maximum quantity that can be transpirated by plant from $R_{1}$, then

$$
T p_{1}(t)=T p_{1 \max }(t)=E T_{0}(t) K_{c}(t) C_{p}(t)
$$

if $R_{1}(t)>0$ and $T_{p 1}(t)=T p_{1 \max }(t)=0$ otherwise. $E T_{0}$ is the reference evapotranspiration determined according to the meteorological conditions ([20]), $K_{c}(t)=K_{c \max }(1-\exp (-L A I(t)))$ the crop coefficient, and $C_{p}(t)=1-\exp (-k L A I(t))$ the partitioning coefficient between plant transpiration and soil evaporation. Both coefficients are related to $L A I$ which is evaluated dynamically by GreenLab. As for the soil evaporation, we have

$$
E s(t)=E T_{0}(t)\left(1-C_{p}(t)\right)
$$

if $R_{1}(t)>0$ and $E s(t)=0$ otherwise.

\subsubsection{Transpiration from $R_{2}$ and hydric stress}

Whenever the water contained in $R_{1}$ is exhausted by evapotranspiration, no more soil evaporation takes place and the plant transpirates in $R_{2}$ if it contains available water. Moreover, in this case we take into account a possible water stress when the volumetric soil water content gets below the threshold value defined as $\Theta_{s}=\Theta_{U}-\Theta_{F U}$, where $\Theta_{U}=\Theta_{\max }-\Theta_{\min }$ is the useful capacity and $\Theta_{F U}=\alpha \Theta_{U}$ is the easily useful capacity, with the fixed ratio $\alpha$ proposed by [5]. The quantity transpirated from $R_{2}$ is

$$
T p_{2}(t)=T p_{2 \max }(t) \min \left(\frac{\Theta_{2}(t)-\Theta_{\min }}{\Theta_{s}}, 1\right)
$$

with

$$
T p_{2 \max }(t)=E T_{0}(t) K_{c}(t)
$$

if $R_{1}(t)=0$ and $R_{2}(t)>0, T p_{2 \max }(t)=0$ otherwise. Finally, the water stress index is obtained as the ratio of the actual transpiration over the maximum transpiration:

$$
f_{s}(t)=\frac{T p_{1}(t)+T p_{2}(t)}{T p_{1 \max }(t)+T p_{2 \max }(t)}
$$

\subsection{A delay system}

The coupled model is a delay system written as

$$
d x(t)=F(t, x(t), x(\eta(t)), u(t))
$$



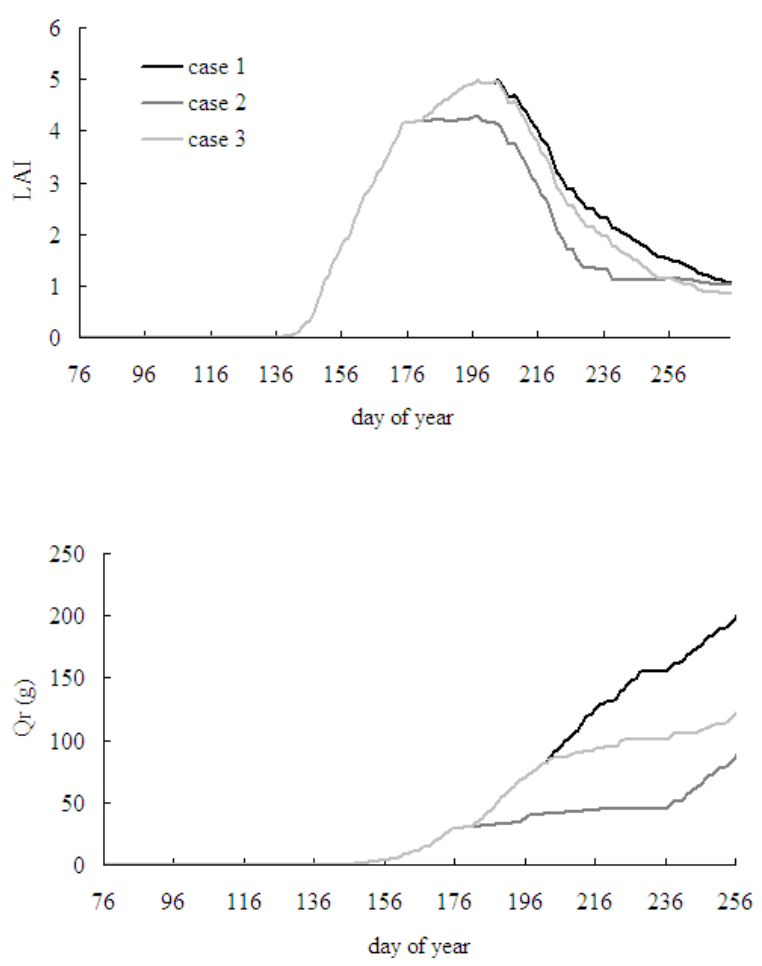

Figure 2. Simulations of LAI and accumulated root mass of sugar beet. Case 1: with real hydric data; case 2: no water input from Day Of Year $(\mathrm{DOY})=156$ to $\mathrm{DOY}=216$; case 3: no water input from $\mathrm{DOY}=179$ to $\mathrm{DOY}=276$.

with the state vector

$$
\mathbf{x}=\left(\mathbf{Q}_{\mathbf{b}}, \Theta_{1}, \Theta_{2}, \Theta_{3}\right)
$$

$u=I$ is the control variable. As later we would like to harmonize the boundary and discretization of state variable, the state components for soil water status are chosen as $\Theta_{i}$, which can be easily converted from eq. 11 .

Precisely, we have eq. 8 and

$$
\begin{aligned}
d \Theta_{1}(t) & =f_{1}\left(t, Q_{b}(t), \Theta_{1}(t), u(t)\right) \\
d \Theta_{2}(t) & =f_{2}\left(t, Q_{b}(t), \Theta_{1}(t), \Theta_{2}(t), \Theta_{3}(t)\right) \\
d \Theta_{3}(t) & =f_{3}\left(t, \Theta_{2}(t), \Theta_{3}(t)\right)
\end{aligned}
$$

$f_{1}, f_{2}, f_{3}$ are all known as explained in section 2.2.

We may solve this delay system interval after interval by using the Euler's method with a constant time step for example ([1],[13]).

\subsection{Simulation}

In this coupling approach, the interaction between plant functional development and the dynamics of soil water balance have strong interactions. This is particularly illustrated by the the LAI simulation. As a crucial index of the plant growth stage, the LAI is used to update the soil water balance, since the quantity evapotranspirated is determined by the crop coverage on the field. In return, the stress level resulting from the soil water balance constrains the dry matter accumulation of plant, and affect accordingly the LAI evolution.

To illustrate this dynamic plant-soil interaction conditioned by water status, we apply the coupled model to sugar beet growth under three hydric condition. All parameters of crop and soil (see [12] for GreenLab parameters) are identical in the three simulations, as well as the input of radiation. One simulation is carried out with real data of rainfall and irrigation, plant suffers almost no hydric stress in this case. In the other two simulations, we introduce some water stress at different period by removing water input in these periods (see Fig.2). Simulations of root biomass under the three hydric condition is also presented. The simulation of biomass evolution by organ compartments show that this coupling approach permits to illustrate the impact of water stress at different periods on crop produtcion.

\section{Optimization of irrigation scheduling}

\subsection{Objective and dynamic programming}

Various objectives may be considered for determining "optimal" irrigation strategies. As suggested by Jensen and al.([8]), a motivating goal for farmers to adopt a nontraditional irrigation practice would be the profit maximization, realized as a balance between the reduction of production cost and the increase of yield. Such an objective can be easily formulated within the framework of the plant-soil model described above. We define firstly the instantaneous profit function as

$$
g(t, \mathbf{x}, \mathbf{u})=a \frac{\bar{P}_{c}}{\bar{D}}(t) q(t)-c(t) u(t)
$$

where $\frac{\bar{P}_{c}}{D}(t) q(t)$ is the part of biomass allocated to the harvest compartment (as cob for maize and root for sugar beet), notice that $q(t)$ is actually a function of the sate vector $x(t) . a$ denotes the unit price of yield and $c(t)$ the unit price of water (possibly varying with time). Therefore, the objective is to maximize the total profit

$$
\int_{0}^{t_{f}} g(t, \mathbf{x}, \mathbf{u}) d t
$$

$t_{f}$ is fixed as the harvest time since plant's initiation. Without loss of relevance to irrigation practice, the control variable can be defined constant per interval, thus we divide the interval $\left[0, t_{f}\right]$ into $N$ subintervals $\left[0, t_{1}\left[,\left[t_{1}, t_{2}[, \ldots\right.\right.\right.$, $\left[t_{N-1}, t_{N}\right]$, with $t_{N}=t_{f}$. In what follows, we take daily time steps, i.e. $t_{n}=n$. And for all $t_{n-1} \leqslant t<t_{n}$,

$$
c(t)=c\left(t_{n-1}\right)=c_{n-1}, u(t)=u\left(t_{n-1}\right)=u_{n-1}
$$


Therefore, if we denote $J^{*}\left(x_{0}\right)$ the optimal profit for a given initial state $x_{0}$, then

$$
J^{*}\left(x_{0}\right)=\max _{u_{0}, u_{1}, \ldots u_{N-1}} \sum_{n=1}^{N} \int_{t_{n-1}}^{t_{n}} g\left(t, x(t), u_{n-1}\right) d t
$$

Previous attempts to determine optimal irrigation strategy of a simplified plant-soil interaction model relied on variational formulation [24] but remained unsuccessful due to the non-convexity of the problem. However, the formulation (28) suggests naturally to apply the dynamic programming (DP). Namely, we have for every $x_{0}, J^{*}\left(x_{0}\right)=$ $J_{0}\left(x_{0}\right)$, with $J_{0}$ obtained by the next stage of the backward algorithm: For all $y \in \mathbb{R}_{+}^{4}$,

$$
J_{N}(y)=0
$$

and

$$
\begin{aligned}
J_{n}(y)= & \max _{u_{n} \in\left[0, \bar{u}_{n}\right]}\left\{\int_{t_{n}}^{t_{n+1}} g\left(t, x_{y}(t), u_{n}\right) d t\right. \\
& \left.+J_{n+1}\left(x_{y}\left(t_{n}\right)+\int_{t_{n}}^{t_{n}+1} f\left(t, x_{y}(t), x_{y}\left(\eta_{t}\right), u_{n}\right) d t\right)\right\}
\end{aligned}
$$

for $n=N-1, \ldots, 0$, where $x_{y}:\left[t_{n}, t_{f}\right] \rightarrow \mathbb{R}_{+}^{4}$ is the solution of eq.20 such that $x_{y}\left(t_{n}\right)=y$.

\subsection{Iterative Dynamic Programming}

However, a direct implementation of dynamic programming is not feasible here, due to the delay term of the system (see eq.20). Trajectories may be simulated previously with different irrigation policy to approximate the delay state value when proceeding the backward algorithm of DP (a trajectory is a finite sequence $x_{0}, \ldots, x_{N}$ as numerical resolution of (see eq. 20). But then, we will confront the "curse of dimensionality" both for the policy space in such a precalculated trajectory procedure and for the state space in the DP algorithm.

The iterative dynamic programming (IDP) is a variant of the standard approach allowing to come across these difficulties ([15], [3]). By IDP, the problem is solved in a series of iterations rather than in a single pass of the DP algorithm. At the beginning of every iteration of IDP, a control grid (resp. state grid) is generated by perturbing the optimal policy (resp. the trajectory with the optimal policy) obtained at the previous iteration and by applying a systematic region contraction. The same treatment is applied to the pre-computation of trajectories to approximate the delay state value.

With this iterative approach, we may just generate relatively coarse grids and a few number of precalculated trajectories at every iteration, since the computation of the global optimal solution actually relies on the convergence of solutions by iteration.

This irrigation optimization procedure is illustrated in Fig. 3 with the coupled model applied to sugar beet. Total

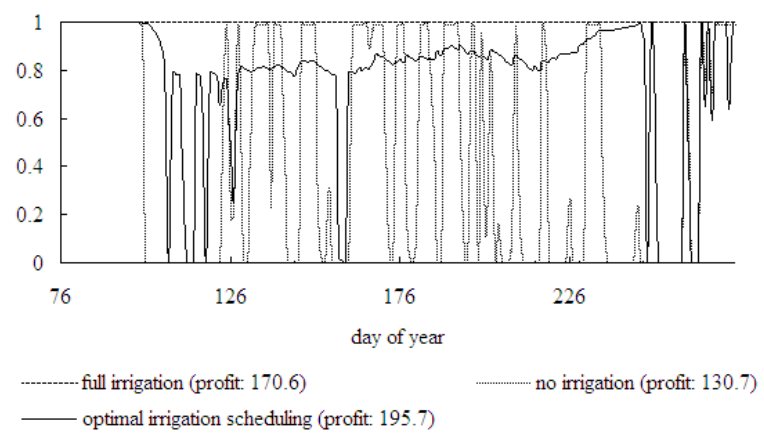

Figure 3. Water stress index for different irrigation strategies

profit (eq.26) and water stress index evolution (eq.19) are compared with the optimal irrigation scheduling and two extreme irrigation strategies.

\section{Discussion and Conclusion}

A new plant-soil model-based irrigation scheduling method is presented in this article. We use a coupled model to simulate the plant-soil interaction, where the plant biomass production is affected by the soil water status.

The impact of water stress on plant structuralfunctional growth used for this study is simplified for the sake of clarity. Generally, other more complex ecophysiological sub-models taking into account the impact of water stress could be integrated, for example the impact on leaf senescence ([19],[21]) or on the rate of leaf appearance([11]). These model refinement will result in varying leaf life spans, phyllochrons and technical adaptations from a numerical point of view, that can be overcome without major difficulty.

The motivation and benefit of our approach of describing integrally the plant-soil interaction is to relate the irrigation strategy directly to the plant growth condition, and to facilitate the use of mathematical optimization method. Dynamic programming shows particularly adapted to find optimal irrigation strategies. For numerical resolution, the iterative version of dynamic programming is adopted to solve the curse of dimensionality and the delay term due to blade senescence.

The next step of our research will be the extension of the introduced framework to the stochastic case. We may consider random inputs like weather data, yield and water prices in the model to maximize the expected value of net return, as well as quantifying the risk levels for farmers.

\section{References}

[1] C. Baker, C. Paul, and D. Wille. Issues in the numerical solution of evolutionary delay differential equa- 
tions. Numerical Analysis 248, University of Manchester, Department of Mathematics, 1999.

[2] P.-H. Cournède, M.-Z. Kang, A. Mathieu, J.-F. Barczi, H.-P. Yan, B.-G. Hu, and P. de Reffye. Structural Factorization of Plants to Compute their Functional and Architectural Growth. Simulation, 82(7):427438, 2006.

[3] S. Dadebo and R. Luus. Optimal control of time-delay systems by dynamic programming. Optimal Control Applications and Methods, 13(1):29-41, 1992.

[4] P. de Reffye, E. Heuvelink, D. Barthélémy, and P.-H. Cournède. Plant growth models. In S.E. Jorgensen and B. Fath, editors, Ecological Models. Vol. 4 of Encyclopedia of Ecology (5 volumes), pages 2824-2837. Elsevier (Oxford), 2008.

[5] J. Doorenbos and A.H. Kassam. Yield response to water. In Food and Agriculture Organisation, Rome, Italy, 1979.

[6] T.C. Hsiao. Plant responses to water stress. Annual review of plant physiology, 24(1):519-570, 1973.

[7] T.C. Hsiao and E. Acevedo. Plant responses to water deficits, water-use efficiency, and drought resistance. Agricultural Meteorology, 14(1-2):59-84, 1974.

[8] M.E. Jensen, D.C.N. Robb, and C.E. Franzoy. Scheduling irrigations using climate-crop-soil data. Proceedings of the American Society of Civil Engineers, Journal of the Irrigation and Drainage Division, 96(IRI):25-38, 1970.

[9] H.G. Jones. Irrigation scheduling: advantages and pitfalls of plant-based methods. Journal of Experimental Botany, 55(407):2427, 2004.

[10] M.R. Khaledian, J.C. Mailhol, P. Ruelle, and P. Rosique. Adapting Pilote model for water and yield management under direct seeding system: The case of corn and durum wheat in a mediterranean context. Agricultural Water Management, 96(5):757 770, 2009.

[11] E. Lebon, A. Pellegrino, G. Louarn, and J. Lecoeur. Branch development controls leaf area dynamics in grapevine (Vitis vinifera) growing in drying soil. Annals of Botany, 98(1):175, 2006.

[12] S. Lemaire, F. Maupas, P.-H. Cournède, and P. de Reffye. A morphogenetic crop model for sugarbeet (beta vulgaris l.). In International Symposium on Crop Modeling and Decision Support: ISCMDS 2008, April 19-22, 2008, Nanjing, China, 2008.

[13] Z.P. Li, V. Le Chevalier, and P.-H. Cournède. Towards a continuous approach of functional-structural plant growth. In B.-G. Li, M. Jaeger, and Y. Guo, editors, 3rd international symposium on Plant Growth and Applications(PMA09), Beijing, China. IEEE, November 9-12 2009. to appear.

[14] J.I. Lizaso, W.D. Batchelor, and M.E. Westgate. A leaf area model to simulate cultivar-specific expansion and senescence of maize leaves. Field Crops Research, 80, 2003.

[15] R. Luus. Iterative dynamic programming. CRC Press, 2000.

[16] G.S. Mac Master and W.W. Wilhelm. Growing degree-days: one equation, two interpretations. Agricultural and Forest Meteorology, 87(4):291-300, 1998.

[17] J.C. Mailhol, A. Olufayo, and P. Ruelle. Sorghum and sunflower evapotranspiration and yield from simulated leaf area index. Agri. Water Manag., 35:167$182,1997$.

[18] JC Mailhol, O. Olufayo, and P. Ruelle. Aet and yields assessments based on the lai simulation. application to sorghum and sunflower crops. Agric Water Manag, 35:167-182, 1997.

[19] S. Munné-Bosch and L. Alegre. Die and let live: leaf senescence contributes to plant survival under drought stress. Functional Plant Biology, 31(3):203216, 2004

[20] H.L. Penman. Natural evaporation from open water, bare soil and grass. Proceedings of the Royal Society of London. Series A, Mathematical and Physical Sciences, 193:120-145, 1948.

[21] F. Tardieu. Drought perception by plants Do cells of droughted plants experience water stress? Plant Growth Regulation, 20(2):93-104, 1996.

[22] C. Varlet-Grancher, G. Gosse, M. Chartier, H. Sinoquet, R. Bonhomme, and J.-M. Allirand. Mise au point : rayonnement solaire absorbé ou intercepté par un couvert végétal. Agronomie, 9:419-439, 1989.

[23] WW Wilhelm, G.S. McMaster, and ARS USDA. Importance of the phyllochron in studying in development and growth in grasses. 1995.

[24] L. Wu, P. De Reffye, B.G. Hu, F.-X. Le Dimet, and P.H. Cournède. A water supply optimization problem for plant growth based on greenlab model. ARIMA, 3:194-207, 2005.

[25] H.P. Yan, M.Z. Kang, P. De Reffye, and M. Dingkuhn. A dynamic, architectural plant model simulating resource-dependent growth. Annals of Botany, 93:591-602, 2004. 